\title{
FUZZY NUMERICAL RANGE HILBERT OPERATORS WITH APPLICATIONS IN BEST APPROXIMATION
}

\author{
M. Iranmanesh ${ }^{1}$, F. Soleimany ${ }^{2} \S$ \\ 1,2 Department of Mathematical Sciences \\ Shahrood University of Technology \\ IRAN
}

\begin{abstract}
The main purpose of this paper is to introduce the fuzzy numerical range of operator on fuzzy Hilbert space and to study some its properties. Then by applying this concept, we study a version of the problem of the best approximation in fuzzy Hilbert operator spaces.
\end{abstract}

AMS Subject Classification: 46S40, 41A50, 03E72

Key Words: fuzzy real number, fuzzy norm, fuzzy bounded linear operator, best approximation

\section{Introduction}

In studying fuzzy topological vector spaces, Katsaras [11] in 1984, first introduced the notion of fuzzy norm on a linear space. Felbin [5] introduced an idea of a fuzzy norm on a linear space by assigning a fuzzy real number to each element of the linear space so that the corresponding metric associated this fuzzy norm is of Kaleva type [10] fuzzy metric. In 1994, Cheng and Mordeson [3] introduced another idea of a fuzzy norm on a linear space in such a manner that the corresponding fuzzy metric of it is of Kramosil and Michelek type [12]. Other papers $[6,7,13]$ have appeared to study various properties of these types of fuzzy normed linear spaces.

Received: June 16, 2016

(C) 2016 Academic Publications

${ }^{\S}$ Correspondence author 
Felbin introduced an idea of fuzzy bounded linear operators over fuzzy normed linear spaces and defined fuzzy norm for such an operator. In this paper we use of the definition of fuzzy inner product and a new norm of on operator introduced by M. Saheli, A. Hasankhani and A. Nazari in [9]. They considered the norm of the operator defined in [1] by T. Bag and S.K. Samanta. We introduce the notions of numerical range of linear operators on fuzzy Hilbert spaces and show some properties them. Veeramani [17], Vaezpour and Karimi [16] introduced the concept of t-best approximations in fuzzy metric spaces and fuzzy normed spaces. In this context we investigate another kind of best approximations in fuzzy bounded linear operator spaces over fuzzy Hilbert spaces.

\section{Preliminaries}

In this section some definitions and preliminary results are given which will be used in our paper.

Definition 1. A mapping $\eta: R \rightarrow[0,1]$ is called a fuzzy real number with $\alpha$-level set $[\eta]_{\alpha}=\{t: \eta(t) \geq \alpha\}$, if it satisfies the following conditions:

$\left(N_{1}\right)$ there exists $t_{0} \in R$ such that $\eta\left(t_{0}\right)=1$.

$\left(N_{2}\right)$ for each $\alpha \in(0,1]$, there exist real numbers $\eta_{\alpha}^{-} \leq \eta_{\alpha}^{+}$such that the $\alpha$-level set $[\eta]_{\alpha}$ is equal to the closed interval $\left[\eta_{\alpha}^{-}, \eta_{\alpha}^{+}\right]$.

The set of all fuzzy real numbers is denoted by $F(R)$. Since each $r \in R$ can be considered as the fuzzy real number $\tilde{r} \in F(R)$ defined by

$$
\tilde{r}(t)=\left\{\begin{array}{ll}
1 & \text { if } t=r \\
0 & \text { if } t \neq r
\end{array},\right.
$$

it follows that $R$ can be embedded in $F(R)$.

Lemma 2. $\eta \in F(R)$ if and only if $\eta: R \rightarrow[0,1]$ satisfies

(i) $\eta$ is normal, i.e. there exists an $x_{0} \in R$ such that $\eta\left(x_{0}\right)=1$

(ii) $\eta$ is fuzzy convex, i.e. $\eta(\alpha x+(1-\alpha) y) \geq \min \{\eta(x), \eta(y)\}$ for all $x, y \in R$ and for all $\alpha \in(0,1)$

(iii) $\eta$ is upper semi-continuous. 
(iv) $\lim _{t \rightarrow-\infty} \eta(t)=0, \lim _{t \rightarrow+\infty} \eta(t)=0$

Definition 3. [10] The arithmetic operations $\oplus, \ominus, \times$ and $\backslash$ on $F(R) \times F(R)$ are defined by

i) $(\eta \oplus \gamma)(t)=\sup _{t=s+h} \min \{\eta(s), \gamma(h)\}$.

ii) $(\eta \times \gamma)(t)=\sup _{t=s \times h} \min \{\eta(s), \gamma(h)\}$.

iii) $(\eta \ominus \gamma)(t)=\sup _{t=s-h} \min \{\eta(s), \gamma(h)\}$.

v) $\left(\frac{\eta}{\gamma}\right)(t)=\sup _{t=\frac{s}{h}} \min \{\eta(s), \gamma(h)\}$.

In this paper, we denote the symbol $\eta^{2}$ to replace $\eta \times \eta$.

Definition 4. [10] Let $\eta \in F(R)$. If $\eta(t)=0$ for all $t<0$, then $\eta$ is called a positive fuzzy real number. The set of all positive fuzzy real numbers is denoted by $F^{+}(R)$, and real number $\eta_{\alpha}^{-} \geq 0$ for all $\eta \in F^{+}(R)$ and all $\alpha \in(0,1]$.

Lemma 5. [10] Let $\eta, \gamma \in F(R)$ and $[\eta]_{\alpha}=\left[\eta_{\alpha}^{-}, \eta_{\alpha}^{+}\right],[\gamma]_{\alpha}=\left[\gamma_{\alpha}^{-}, \gamma_{\alpha}^{+}\right]$. Then,

i) $[\eta \oplus \gamma]_{\alpha}=\left[\eta_{\alpha}^{-}+\gamma_{\alpha}^{-}, \eta_{\alpha}^{+}+\gamma_{\alpha}^{+}\right]$;

ii) $[\eta \ominus \gamma]_{\alpha}=\left[\eta_{\alpha}^{-}-\gamma_{\alpha}^{+}, \eta_{\alpha}^{+}-\gamma_{\alpha}^{-}\right]$;

iii) $[\eta \otimes \gamma]_{\alpha}=\left[\eta_{\alpha}^{-} \gamma_{\alpha}^{-}, \eta_{\alpha}^{+} \gamma_{\alpha}^{+}\right]$for $\eta, \gamma \in F^{+}(R)$;

v) $\left[\frac{\tilde{1}}{\eta}\right]_{\alpha}=\left[\frac{1}{\eta_{\alpha}^{+}}, \frac{1}{\eta_{\alpha}^{-}}\right]$, if $\eta_{\alpha}^{-}>0$.

Lemma 6. [10] Let $\left[a^{\alpha}, b^{\alpha}\right], \alpha \in(0,1]$, be a given family of non-empty intervals. Suppose

(a) for all, $0<\alpha_{1} \leq \alpha_{2},\left[a^{\alpha_{2}}, b^{\alpha_{2}}\right] \subseteq\left[a^{\alpha_{1}}, b^{\alpha_{1}}\right]$;

(b) for any increasing sequence $\left\{\alpha_{k}\right\}$ in $(0,1]$ converging to $\alpha$,

$$
\left[\lim _{k \rightarrow \infty} a^{\alpha_{k}}, \lim _{k \rightarrow \infty} b^{\alpha_{k}}\right]=\left[a^{\alpha}, b^{\alpha}\right]
$$

(c) $-\infty<a^{\alpha} \leq b^{\alpha}<+\infty$ for all $\alpha \in(0,1]$. 
Then the family $\left[a^{\alpha}, b^{\alpha}\right]$ represents the $\alpha$-level sets of a fuzzy real number $\eta$ in $F(R)$. Conversely, if $\left[a^{\alpha}, b^{\alpha}\right], \alpha \in(0,1]$ are $\alpha$-level sets of a fuzzy real number $F(R)$, then the conditions (a), (b) and (c) are satisfied.

Definition 7. [10] The absolute value $|\eta|$ of $F(R)$ is defined by

$$
|\eta|(t)=\max \{\eta(t), \eta(-t)\} .
$$

Definition 8. [9] For a positive fuzzy real number $\eta$ define $\sqrt{\eta}=\gamma$, where $[\gamma]_{\alpha}=\left[\sqrt{\eta_{\alpha}^{-}}, \sqrt{\eta_{\alpha}^{+}}\right]$.

Definition 9. [9] The sequence $\left\{\eta_{n}\right\}$ in $F(R)$ converges to $\eta$ in $F(R)$, if $\lim _{n \rightarrow \infty}\left|\eta_{n}-\eta\right| \alpha^{+}=0$.

Definition 10. [10] Let $\eta, \delta \in F(R)$ and $[\eta]_{\alpha}=\left[\eta_{\alpha}^{-}, \eta_{\alpha}^{+}\right],[\delta]_{\alpha}=\left[\delta_{\alpha}^{-}, \delta_{\alpha}^{+}\right]$. Define a partial ordering by $\eta \leq \delta$, if and only if $\eta_{\alpha}^{-} \leq \delta_{\alpha}^{-}, \eta_{\alpha}^{+} \leq \delta_{\alpha}^{+}$for all $\alpha \in(0,1]$.

Definition 11. A subset $A$ of $F(R)$ is said to be bounded from above if there exists a fuzzy real number $\eta$, called an upper bound of $A$, such that $\nu \leq \eta$ for every $\nu \in A$. $\mathrm{u}$ is called the least upper bound or supremum of $\mathrm{A}$ if $u$ is an upper bound and $\eta \leq \eta_{i}$ for all upper bounds $\eta_{i}$. Similarly, a lower bound and the greatest lower bound or infimum are defined. $A$ is said to be bounded, if it is both bounded from above and below.

Definition 12. [18] Let $X$ be a vector space over $R$. Assume the mappings $L ; R:[0,1] \times[0,1] \rightarrow[0,1]$ are symmetric and non-decreasing in both arguments, and that $L(0 ; 0)=0$ and $R(1 ; 1)=1$. Let $\|\|:. X \rightarrow F^{+}(R)$. The quadruple $(X ;\|\| ; L ; R$.$) is called a fuzzy normed linear space (briefly, FNS) with the fuzzy$ norm $\|$.$\| , if the following conditions are satisfied:$

$\left(F_{1}\right)$ if $x \neq 0$ then $\inf _{0<\alpha \leq 1}\|x\|_{\alpha}^{-}>0$,

$\left(F_{2}\right)$ if $\|x\|=\tilde{0}$ if and only if $x=0$,

$\left(F_{3}\right)\|r x\|=\tilde{r}\|x\|$, for $x \in X, r \in R$,

$\left(F_{4}\right)$ for $x, y \in X$,

$\left(F_{4} L\right)\|x+y\|(s+t) \geq L(\|x\|(s) ;\|x\|(t))$ whenever $s \leq\|x\|_{1}^{-}, t \leq\|x\|_{1}^{-}$ and $s+t \leq\|x+y\|_{1}^{-}$, 
$\left(F_{4} R\right)\|x+y\|(s+t) \leq R(\|x\|(s) ;\|x\|(t))$ whenever $s \geq\|x\|_{1}^{-}, t \geq\|x\|_{1}^{-}$ and $s+t \geq\|x+y\|_{1}^{-}$.

Lemma 13. [18] Let $(X ;\|\| ; L ; R$.$) be an FNS.$

(1) If $L \leq \min$, then $F_{4} L$ holds whenever $\|x+y\|_{\alpha}^{-} \leq\|x\|_{\alpha}^{-}+\|x\|_{\alpha}^{-}$, for all $x, y \in X$ and $\alpha \in(0,1]$.

(2) If $L \geq \min$, then $\|x+y\|_{\alpha}^{-} \leq\|x\|_{\alpha}^{-}+\|x\|_{\alpha}^{-}$, for all $x, y \in X$ and $\alpha \in(0,1]$, whenever $F_{4} L$ holds.

(3) If $R \leq \max$, then $F_{4} R$ holds whenever $\|x+y\|_{\alpha}^{+} \leq\|x\|_{\alpha}^{+}+\|x\|_{\alpha}^{+}$, for all $\alpha \in(0,1]$.

(4) If $R \geq \max$, then $\|x+y\|_{\alpha}^{-} \leq\|x\|_{\alpha}^{-}+\|x\|_{\alpha}^{+}$, for all for all $x, y \in X$ and $\alpha \in(0,1]$, whenever $F_{4} R$ holds.

In the sequel we fix $L(s, t)=\min (s, t)$ and $R(s, t)=\max (s, t)$ for all $s, t \in$ $[0,1]$ and we write $(X,\|\|$.$) or simply X$ when $L$ and $R$ are as indicated above. The following result is an analogue of the triangle inequality.

Theorem 14. [18] In a fuzzy normed linear space $(X ;\|\|$.$) , the condition$ $F_{4}$ is equivalent to

$$
\|x+y\| \leq\|x\| \oplus\|y\|
$$

Definition 15. [18] Let $(X,\|\|)$ be a FNS. A sequence $x_{n} \in X$ is said to converge to $x \in X\left(\lim _{n \rightarrow \infty} x_{n}=x\right)$, if $\lim _{n \rightarrow \infty}\left\|x_{n}-x\right\|_{\alpha}^{+}=0$ for all $\alpha \in(0,1]$.

The function $f: X \longrightarrow F(R)$ is called fuzzy convex if for each $x, y \in X$ and each $\lambda \in(0,1)$,

$$
f(\lambda x+(1-\lambda) y) \leq \tilde{\lambda} f(x)+(1 \stackrel{\sim}{-\lambda}) f(y) .
$$

Definition 16. [2] Let $f: X \rightarrow F(R)$ be a fuzzy mapping. $f$ is upper semi-continuous at a point $x_{0} \in X$ if for any $\varepsilon>0$, there exists a $\delta>0$ such that

$$
F(x) \leq F\left(x_{0}\right)+\tilde{\varepsilon},
$$

for all $x \in X$ and $\left\|x-x_{0}\right\| \leq \delta$. $f$ is said to be upper semi-continuous on $X$ if it is upper semi-continuous at each point of $X$. 
In [14] Yu-Ru Syaua, Ly-Fie Sugiantob, E. Stanley Leec stated that this concept of upper semicontinuity is essentially same as if both $F(x)_{\alpha}^{+}$and $F(x)_{\alpha}^{-}$ are upper semicontinuous at $x_{0}$ uniformly for $\alpha \in(0,1]$.

Definition 17. Suppose $X$ is a fuzzy normed space and $T: X \rightarrow X$ is an operator. The operator $T$ is called fuzzy bounded if for all $x \in X$ we have

$$
\exists k>0,\|T x\| \leq \tilde{k}\|x\| \text {. }
$$

Definition 18. [9] Suppose $T: X \rightarrow X$ is a fuzzy bounded. Define fuzzy operator norm of $T$ in form follows

$$
[\|T\|]_{\alpha}=\left[\sup _{\beta<\alpha} \sup _{\|x\|_{\beta}^{-} \leq 1}\|T x\|_{\beta}^{-}, \inf \left\{\eta_{\alpha}^{+}:\|T x\| \leq \eta\|x\|\right\}\right],
$$

for all $\alpha \in(0,1]$, i.e. $\|T\|_{\alpha}=\left[\|T\|_{\alpha}^{-},\|T\|_{\alpha}^{+}\right]$, where

$$
\begin{gathered}
{[\|T\|]_{\alpha}^{-}=\sup _{\beta<\alpha} \sup _{\|x\|_{\beta}^{-} \leq 1}\|T x\|_{\beta}^{-},} \\
\|T\|_{\alpha}^{+}=\inf \left\{\eta_{\alpha}^{+}: \quad\|T x\| \leq \eta\|x\|\right\} .
\end{gathered}
$$

Definition 19. [9] Let $X$ be a vector space over $R$. A fuzzy inner product on $X$ is a mapping such that for all vectors $x, y, z \in X$ and all $r \in R$, we have:

i) $\langle x, x\rangle=\tilde{0}$ if and only if $x=0$,

ii) $\langle x, y\rangle=\langle y, x\rangle$,

iii) $\langle x+y, z\rangle=\langle x, z\rangle \oplus\langle y, z\rangle$,

v) $\langle r x, y\rangle=\tilde{r}\langle x, y\rangle$, for $x \in X, r \in R$,

iv) for $x \neq 0, \inf _{0<\alpha \leq 1}\langle x, x\rangle_{\alpha}^{-}>0$.

The vector space $X$ equipped with a fuzzy inner product is called a fuzzy inner product space.

Lemma 20. [9] A fuzzy inner product space $X$ together with its corresponding norm ||.|| satisfy the Schwarz inequality

$$
\mid\langle x, y\rangle \leq\|x\| \times\|y\|, \text { for all } x, y \in X .
$$




\section{Main results}

Let $(H,\langle\rangle)$ be a fuzzy Hilbert space with by inner product \langle\rangle . The set of all fuzzy bounded linear operators $T: H \rightarrow H$ is denoted by $B(H)$. At first, we introduce the notions of numerical range of linear operators on fuzzy Hilbert space $H$. Then we give results of best approximation in the space.

Lemma 21. Let $f, g \in B(H)$ and $\left\{x_{n}\right\} \in H$ be such that $\left\|x_{n}\right\|_{\alpha}^{-} \leq 1$, for all $\alpha \in(0,1]$,

$$
\lim _{n \rightarrow \infty}\left\langle f\left(x_{n}\right), g\left(x_{n}\right)\right\rangle_{\alpha}^{-} \text {and } \lim _{n \rightarrow \infty} \frac{\left\langle f\left(x_{n}\right), g\left(x_{n}\right)\right\rangle_{\alpha}^{+}}{\left\|x_{n}\right\|_{\alpha}^{-2}}
$$

exist. Then $\eta^{x_{n}}$, where

$$
\left[\eta^{x_{n}}\right]_{\alpha}:=\left[\sup _{\beta<\alpha} \lim _{n \rightarrow \infty}\left\langle f\left(x_{n}\right), g\left(x_{n}\right)\right\rangle_{\beta}^{-}, \lim _{n \rightarrow \infty} \frac{\left\langle f\left(x_{n}\right), g\left(x_{n}\right)\right\rangle_{\alpha}^{+}}{\left\|x_{n}\right\|_{\alpha}^{-2}}\right]
$$

is a fuzzy real number.

Proof. Since the inner product \langle\rangle is a fuzzy real number and $\frac{1}{\left\|x_{n}\right\|_{\alpha}^{-}} \geq 1$, for $\alpha \in(0,1]$ and $\beta \in(0, \alpha)$ we have

$$
\left\langle f\left(x_{n}\right), g\left(x_{n}\right)\right\rangle_{\beta}^{-} \leq\left\langle f\left(x_{n}\right), g\left(x_{n}\right)\right\rangle_{\beta}^{+} \leq\left\langle f\left(x_{n}\right), g\left(x_{n}\right)\right\rangle_{\alpha}^{+} \leq \frac{\left\langle f\left(x_{n}\right), g\left(x_{n}\right)\right\rangle_{\alpha}^{+}}{\left\|x_{n}\right\|_{\alpha}^{-2}} .
$$

Now by taking lim and sup on $\beta$, we have

$$
\sup _{\beta<\alpha} \lim _{n \rightarrow \infty}\left\langle f\left(x_{n}\right), g\left(x_{n}\right)\right\rangle_{\beta}^{-} \leq \lim _{n \rightarrow \infty} \frac{\left\langle f\left(x_{n}\right), g\left(x_{n}\right)\right\rangle_{\alpha}^{+}}{\left\|x_{n}\right\|_{\alpha}^{-2}},
$$

and thus

$$
\eta_{\alpha}^{x_{n}-} \leq \eta_{\alpha}^{x_{n}+}
$$

Hence $\left[\eta^{x_{n}}\right]_{\alpha}=\left[\eta_{\alpha}^{x_{n}-}, \eta_{\alpha}^{x_{n}+}\right]$ is a nonempty interval, for all $\alpha \in(0,1]$.

(a) Let $0<\alpha_{1} \leq \alpha_{2}$. By Lemma 6 (a) for fuzzy real number \langle\rangle we have

$$
\sup _{\beta<\alpha_{1}} \lim _{n \rightarrow \infty}\left\langle f\left(x_{n}\right), g\left(x_{n}\right)\right\rangle_{\beta}^{-} \leq \sup _{\beta<\alpha_{2}} \lim _{n \rightarrow \infty}\left\langle f\left(x_{n}\right), g\left(x_{n}\right)\right\rangle_{\beta}^{-} .
$$

Also since

$$
\left\|x_{n}\right\|_{\alpha_{1}}^{-2} \leq\left\|x_{n}\right\|_{\alpha_{2}}^{-2} \text { and } \lim _{n \rightarrow \infty}\left\langle f\left(x_{n}\right), g\left(x_{n}\right)\right\rangle_{\alpha_{2}}^{+} \leq \lim _{n \rightarrow \infty}\left\langle f\left(x_{n}\right), g\left(x_{n}\right)\right\rangle_{\alpha_{1}}^{+},
$$

then

$$
\lim _{n \rightarrow \infty} \frac{\left\langle f\left(x_{n}\right), g\left(x_{n}\right)\right\rangle_{\alpha_{2}}^{+}}{\left\|x_{n}\right\|_{\alpha_{2}}^{-2}} \leq \lim _{n \rightarrow \infty} \frac{\left\langle f\left(x_{n}\right), g\left(x_{n}\right)\right\rangle_{\alpha_{1}}^{+}}{\left\|x_{n}\right\|_{\alpha_{1}}^{-2}} .
$$


Then

$$
\left[\eta_{\alpha_{2}}^{x_{n}-}, \eta_{\alpha_{2}}^{x_{n}+}\right] \subseteq\left[\eta_{\alpha_{1}}^{x_{n}-}, \eta_{\alpha_{1}}^{x_{n}+}\right]
$$

(b) Let $\left\{a_{k}\right\}$ be a increasing sequence in $(0,1]$ converging to $\alpha$. Then we have

$$
\begin{aligned}
\lim _{k \rightarrow \infty} \eta_{\alpha_{k}}^{x_{n}+} & =\lim _{k \rightarrow \infty} \lim _{n \rightarrow \infty} \frac{\left\langle f\left(x_{n}\right), g\left(x_{n}\right)\right\rangle_{\alpha}^{+}}{\left\|x_{n}\right\|_{\alpha_{k}}^{2}} \\
& =\lim _{k \rightarrow \infty} \frac{\left\langle\lim _{n \rightarrow \infty} f\left(x_{n}\right), \lim _{n \rightarrow \infty} g\left(x_{n}\right)\right\rangle_{\alpha_{k}}^{+}}{\left\|\lim _{n \rightarrow \infty} x_{n}\right\|_{\alpha_{k}}^{-2}} \\
& =\frac{\left\langle\lim _{n \rightarrow \infty} f\left(x_{n}\right), \lim _{n \rightarrow \infty} g\left(x_{n}\right)\right\rangle_{\alpha}^{+}}{\left\|\lim _{n \rightarrow \infty} x_{n}\right\|_{\alpha}^{-2}} \\
& =\lim _{n \rightarrow \infty} \frac{\left\langle f\left(x_{n}\right), g\left(x_{n}\right)\right\rangle_{\alpha}^{+}}{\left\|x_{n}\right\|_{\alpha}^{-2}} .
\end{aligned}
$$

Hence $\lim _{k \rightarrow \infty} \eta_{\alpha_{k}}^{x_{n}+}=\eta_{\alpha}^{x_{n}+}$. Similarly, we can show that $\lim _{k \rightarrow \infty} \eta_{\alpha_{k}}^{x_{n}-}=\eta_{\alpha}^{x_{n}-}$. Hence,

$$
\left[\lim _{k \rightarrow \infty} \eta_{\alpha_{k}}^{x_{n}-}, \lim _{k \rightarrow \infty} \eta_{\alpha_{k}}^{x_{n}+}\right]=\left[\eta_{\alpha}^{x_{n}-}, \eta_{\alpha}^{x_{n}+}\right] .
$$

(c) By assumtion, for all $\alpha \in(0,1]$,

$$
\lim _{n \rightarrow \infty}\left\langle f\left(x_{n}\right), g\left(x_{n}\right)\right\rangle_{\alpha}^{-} \text {and } \lim _{n \rightarrow \infty} \frac{\left\langle f\left(x_{n}\right), g\left(x_{n}\right)\right\rangle_{\alpha}^{+}}{\left\|x_{n}\right\|_{\alpha}^{-2}}
$$

exist. Therefore,

$$
-\infty<\eta_{\alpha}^{x_{n}-} \leq \eta_{\alpha}^{x_{n}+}<+\infty, \text { for } \alpha \in(0,1] .
$$

Thus by Lemma $6, \eta^{x_{n}}$ is a fuzzy real number.

Suppose $T \in B(H)$. We denote by $Z_{T}$ the following set:

$$
Z_{T}:=\left\{\left\{x_{n}\right\} \in H:\left\|x_{n}\right\|=\widehat{1}, \sup _{\beta<\alpha} \lim _{n \rightarrow \infty}\left\|T x_{n}\right\|_{\beta}^{-}=\|T\|_{\alpha}^{-}\right\} .
$$

Definition 22. The numerical range of $f$ relative to $g$ which is denoted by $W(f g)$ is defined as follows:

$$
W(f g):=\left\{\eta^{x_{n}} \in F(R):\left\{x_{n}\right\} \in Z_{f}\right\} .
$$


Note that $W(f g)$ is non-empty, to see that by the definition of the operator norm that there exists a sequence of unit vectors $\left\{x_{n}\right\} \in H$ such that $\left\{x_{n}\right\} \in$ $Z_{f}$. Therefore, since $\lim _{n \rightarrow \infty}\left\langle f\left(x_{n}\right), g\left(x_{n}\right)\right\rangle_{\alpha}^{-}$and $\lim _{n \rightarrow \infty} \frac{\left\langle f\left(x_{n}\right), g\left(x_{n}\right)\right\rangle_{\alpha}^{+}}{\left\|x_{n}\right\|_{\alpha}^{-2}}$ is a bounded sequence, there exists subsequences $n_{k_{\alpha}}$ that converge to some $\lambda_{\alpha}, \beta_{\alpha} \in R$. Let

$$
\left[\eta^{x_{n_{k_{\alpha}}}}\right]_{\alpha}:=\left[\sup _{\beta<\alpha} \lim _{n_{k_{\alpha}} \rightarrow \infty}\left\langle f\left(x_{n_{k_{\alpha}}}\right), g\left(x_{n_{k_{\alpha}}}\right)\right\rangle_{\beta}^{-}, \lim _{n_{k_{\alpha}} \rightarrow \infty} \frac{\left\langle f\left(x_{n_{k_{\alpha}}}\right), g\left(x_{n_{k_{\alpha}}}\right)\right\rangle_{\alpha}^{+}}{\left\|x_{n_{k_{\alpha}}}\right\|_{\alpha}^{-2}}\right] .
$$

By Lemma $21 \eta^{x_{n_{\alpha}}} \in W(f g)$, and thus $W(f g)$ is non-empty.

Proposition 23. Let $f, g \in B(H)$. Then $W(f g)$ has an upper bound.

Proof. Let $\eta^{x_{n}} \in W(f g)$, for $\alpha \in(0,1]$ and $\beta<\alpha$, from (1) and by Schwarz inequality we have

$$
\left\langle f\left(x_{n}\right), g\left(x_{n}\right)\right\rangle_{\beta}^{-} \leq\left|\left\langle f\left(x_{n}\right), g\left(x_{n}\right)\right\rangle\right|_{\beta}^{-} \leq \|\left\langle f\left(x_{n}\right)\left\|_{\beta}^{-}\right\| g\left(x_{n}\right) \|_{\beta}^{-} .\right.
$$

By taking limits on $n$ and sup on $\beta<\alpha$ we have

$$
\eta_{\alpha}^{x_{n}-} \leq(\|f\| \otimes\|g\|)_{\alpha}^{-} .
$$

Similarly we can show that $\eta_{\alpha}^{x_{n}+} \leq(\|f\| \otimes\|g\|)_{\alpha}^{+}$. Thus $\eta^{x_{n}} \leq\|f\| \otimes\|g\|$ for $\eta^{x_{n}} \in W(f g)$, so $\|f\| \otimes\|g\|$ is a upper bounded for $W(f g)$.

J. Fang, H. Huang, and Talo improved the expressions of the supremum and infimum, limit inferior and limit superior of a bounded sequence of fuzzy real numbers on the concept Goetschel and Voxman [8]. In the following we give the expressions for endpoints of level sets of the supremum and infimum same as that given in $[4,15]$.

Definition 24. Let $A$ be a non-empty subset of $F(R)$. If $A$ has a lower bound, then we define its infimum $\inf (A)$ by

$$
[\inf (A)]_{\alpha}=\left[\sup _{\beta<\alpha} \inf _{\eta \in A} \eta_{\beta}^{-}, \inf _{\eta \in A} \eta_{\alpha}^{+}\right]
$$

for each $\alpha \in(0,1]$. Dually, if $A$ has an upper bound, then its $\operatorname{supremum} \sup (A)$ is defined by

$$
[\sup (A)]_{\alpha}=\left[\sup _{\eta \in A} \eta_{\alpha}^{-}, \inf _{\beta<\alpha} \sup _{\eta \in A} \eta_{\beta}^{+}\right] .
$$

for each $\alpha \in(0,1]$. 
Definition 25. Let $\left\{u_{n}\right\}$ be a bounded sequence of fuzzy real numbers. Then, its $\lim \sup u_{n}=\mu$ has the following expressions:

$$
n \rightarrow \infty
$$

$$
[\mu]_{\alpha}=\left[\limsup _{n \rightarrow \infty} u_{n_{\beta}}^{-}, \inf _{\beta<\alpha} \limsup _{n \rightarrow \infty} u_{n_{\beta}}^{+}\right]
$$

for each $\alpha \in(0,1]$.

Let $f, g \in B(H)$, and $t_{n} \in R$ such that $t_{m} \rightarrow 0^{+}$consider the norm derivatives

$$
\tau_{2}(f, g):=\limsup _{m \rightarrow \infty} \frac{\left\|f+t_{m} g\right\|^{2}-\|f\|^{2}}{2 t_{m}} .
$$

Lemma 26. Let $f, g \in B(H)$. Then,

$$
\tau_{2}(f, g)_{\alpha}^{+} \geq \sup W(f g)_{\alpha}^{+} .
$$

Proof. Suppose $\left\{x_{n}\right\} \in Z_{f}$, we get

$$
\begin{aligned}
& \left\|f+t_{m} g\right\|_{\beta}^{+^{2}} \geq \lim _{n \rightarrow \infty}\left\|f\left(x_{n}\right)+t_{m} g\left(x_{n}\right)\right\|_{\beta}^{+^{2}} \\
& =\lim _{n \rightarrow \infty}\left(\left\|f\left(x_{n}\right)\right\|_{\alpha}^{+^{2}}+t^{2}\left\|g\left(x_{n}\right)\right\|_{\beta}^{+^{2}}+2 t_{m}\left\langle f\left(x_{n}\right), g\left(x_{n}\right)\right\rangle_{\beta}^{+}\right) \\
& =\|f\|_{\beta}^{+^{2}}+t_{m}^{2} \lim _{n \rightarrow \infty}\left\|g\left(x_{n}\right)\right\|_{\beta}^{+^{2}}+2 t_{m} \lim _{n \rightarrow \infty}\left\langle f\left(x_{n}\right), g\left(x_{n}\right)\right\rangle_{\beta}^{+},
\end{aligned}
$$

hence

$$
\frac{\left\|f+t_{m} g\right\|_{\beta}^{+^{2}}-\|f\|_{\beta}^{+^{2}}}{2 t_{m}}=t_{m} \lim _{n \rightarrow \infty}\left\|g\left(x_{n}\right)\right\|_{\beta}^{+^{2}}+\lim _{n \rightarrow \infty}\left\langle f\left(x_{n}\right), g\left(x_{n}\right)\right\rangle_{\beta}^{+},
$$

setting $m \rightarrow \infty$, and taking limsup, then

$$
\limsup _{m \rightarrow \infty} \frac{\left\|f+t_{m} g\right\|_{\beta}^{+^{2}}-\|f\|_{\beta}^{+^{2}}}{2 t_{m}} \geq \lim _{n \rightarrow \infty}\left\langle f\left(x_{n}\right), g\left(x_{n}\right)\right\rangle_{\beta}^{+}=\eta_{\beta}^{x_{n}+}, \text { for }\left\{x_{n}\right\} \in Z_{f} \text {. }
$$

Therefore

$$
\limsup _{m \rightarrow \infty} \frac{\left\|f+t_{m} g\right\|_{\beta}^{+^{2}}-\|f\|_{\beta}^{+^{2}}}{2 t_{m}} \geq \sup _{\left\{x_{n}\right\} \in Z_{f}} \eta_{\beta}^{x_{n}+}
$$

So

$$
\inf _{\beta<\alpha} \limsup _{m \rightarrow \infty} \frac{\left\|f+t_{m} g\right\|_{\beta}^{+^{2}}-\|f\|_{\beta}^{+^{2}}}{2 t_{m}} \geq \inf _{\beta<\alpha} \sup _{\left\{x_{n}\right\} \in Z_{f}} \eta_{\beta}^{x_{n}+}
$$


Since $-\|f\|_{\beta}^{-}>-\|f\|_{\beta}^{+}$, then

$$
\tau_{2}(f, g)_{\alpha}^{+} \geq \sup W(f g)_{\alpha}^{+} .
$$

Theorem 27. Let $U$ be a closed convex subset of $B(H), f \in B(H) \backslash U$, $g_{0} \in U$ and $Z_{f-g_{0}}=\left\{\left\{z_{n}\right\},\left\{-z_{n}\right\}\right\}$. If for each $h \in U$,

$$
\sup W\left(\left(f-g_{0}\right)\left(g_{0}-h\right)\right)_{\alpha}^{-} \geq 0, \text { for } \alpha \in(0,1] .
$$

Then $\left\|f-g_{0}\right\|_{\alpha}^{-}=\inf _{h \in U}\|f-h\|_{\alpha}^{-}$.

Proof. Suppose the inequality (6) holds and let $\left\{x_{n}\right\}=\left\{z_{n}\right\}$ or $\left\{-z_{n}\right\}$. Then for $\alpha \in(0,1]$ we have

$$
\sup _{\beta<\alpha} \lim _{n \rightarrow \infty}\left\langle\left(f-g_{0}\right)\left(x_{n}\right),\left(g_{0}-h\right)\left(x_{n}\right)\right\rangle_{\beta}^{-} \geq 0 .
$$

The (7) implies that $\lim _{n \rightarrow \infty}\left\langle\left(f-g_{0}\right)\left(x_{n}\right),\left(g_{0}-h\right)\left(x_{n}\right)\right\rangle_{\beta}^{-} \geq 0$ because if there exists $\beta_{0}$ such that $\lim _{n \rightarrow \infty}\left\langle\left(f-g_{0}\right)\left(x_{n}\right),\left(g_{0}-h\right)\left(x_{n}\right)\right\rangle_{\beta_{0}}^{-}<0$. Then for $\lambda \in\left(0, \beta_{0}\right]$ we have

$$
\lim _{n \rightarrow \infty}\left\langle\left(f-g_{0}\right)\left(x_{n}\right),\left(g_{0}-h\right)\left(x_{n}\right)\right\rangle_{\lambda}^{-} \leq \lim _{n \rightarrow \infty}\left\langle\left(f-g_{0}\right)\left(x_{n}\right),\left(g_{0}-h\right)\left(x_{n}\right)\right\rangle_{\beta_{0}}^{-}<0 .
$$

Therefore

$$
\sup _{\lambda<\beta_{0}} \lim _{n \rightarrow \infty}\left\langle\left(f-g_{0}\right)\left(x_{n}\right),\left(g_{0}-h\right)\left(x_{n}\right)\right\rangle_{\beta}^{-}<0 .
$$

But it is a contradiction with $(7)$ is true for $\alpha \in(0,1]$. Therefore we have

$$
\begin{aligned}
\|f-h\|_{\alpha}^{-2} & \geq \sup _{\beta<\alpha} \lim _{n_{k} \rightarrow \infty}\left\|f\left(x_{n_{k}}\right)-h\left(x_{n_{k}}\right)\right\|_{\beta}^{-2} \\
& =\sup _{\beta<\alpha} \lim _{n_{k} \rightarrow \infty}\left\|f\left(x_{n_{k}}\right)-g_{0}\left(x_{n_{k}}\right)+g_{0}\left(x_{n_{k}}\right)-h\left(x_{n_{k}}\right)\right\|_{\beta}^{-2} \\
& =\sup _{\beta<\alpha}\left[\lim _{n_{k} \rightarrow \infty}\left\|f\left(x_{n_{k}}\right)-g_{0}\left(x_{n_{k}}\right)\right\|_{\beta}^{-2}+\left\|g_{0}\left(x_{n_{k}}\right)-h\left(x_{n_{k}}\right)\right\|_{\beta}^{-2}\right] \\
& \left.+2\left\langle\left(f-g_{0}\right)\left(x_{n_{k}}\right), g_{0}\left(x_{n_{k}}\right)-h\left(x_{n_{k}}\right)\right\rangle_{\beta}^{-}\right) \\
& \geq \sup _{\beta<\alpha} \lim _{n_{k} \rightarrow \infty}\left\|f\left(x_{n_{k}}\right)-g_{0}\left(x_{n_{k}}\right)\right\|_{\beta}^{-2}=\left\|f-g_{0}\right\|_{\alpha}^{-2} .
\end{aligned}
$$

Then $\|f-h\|_{\alpha}^{-} \geq\left\|f-g_{0}\right\|_{\alpha}^{-}$for $h \in U$. 
Theorem 28. Let $U$ be a closed convex subset of $B(H), f \in B(H) \backslash U$ and $g_{0} \in U$. If $\left\|f-g_{0}\right\|_{\alpha}^{-}=\inf _{h \in U}\|f-h\|_{\alpha}^{-}$for $\alpha \in(0,1]$. Then for each $h \in U$, $\tau_{2}\left(f-h, h-g_{0}\right)_{\alpha}^{-} \leq 0$.

Proof. $i \rightarrow i i$. Let $k, l \in B(H)$, then from Lemma 5 we have

$$
\left(\left\|\frac{b+l}{2}\right\|^{2} \leq\left[\left\|\frac{b}{2}\right\| \oplus\left\|\frac{l}{2}\right\|\right]^{2} \leq \frac{\|b\|^{2} \oplus\|l\|^{2}}{\tilde{2}} .\right.
$$

With the help of the induction method we have

$$
\left(\left\|\frac{b_{1}+\ldots+b_{2^{k}}}{2^{k}}\right\|^{2} \leq \frac{\left\|b_{1}\right\|^{2} \oplus \ldots \oplus\left\|b_{2^{k}}\right\|^{2}}{2^{k}},\right.
$$

now let $b_{1}=\ldots=b_{n}=b$ and $b_{2^{k}-n}=\ldots=b_{2^{k}}=l$, then

$$
\left(\frac{\| n b+\left(2^{k}-n\right) l}{2^{k}} \|^{2} \leq \frac{\tilde{n}\|b\|^{2}}{2^{\tilde{k}}} \oplus \frac{\left(2^{k} \tilde{-} n\right)\|l\|^{2}}{\tilde{2^{k}}} .\right.
$$

Since $A=\left\{\frac{n}{2^{k}}: k \in K\right\}$ is dense on $[0,1]$, so for $\lambda \in(0,1]$ we have $\frac{\tilde{n}}{2^{k}} \rightarrow \tilde{\lambda}$, therefore

$$
\|\lambda b+(1-\lambda) l\|^{2} \leq \tilde{\lambda}\|b\|^{2} \oplus(1 \stackrel{\sim}{\sim} \lambda)\|l\|^{2} .
$$

Then $\|\cdot\|^{2}$ is a fuzzy convex function. The function $\phi$ defined by $\phi(t)=\frac{\|t g\|^{2}}{t}$ is non-decreasing. Because if we put $l=0$ from (9), for $s, t \in R$ such that $0<s \leq t$, we have

$$
\left(\|s b\|^{2} \leq \frac{\tilde{s}}{t}\|t b\|^{2} \oplus \frac{(t \tilde{\sim} s)}{t}\|0\|^{2} .\right.
$$

Since $\|0\|=\tilde{0}$, then $\phi(s) \leq \phi(t)$. Now apply this argument to show that the function $\phi(t)=\frac{\|f+t g\|^{2}-\|f\|^{2}}{\tilde{t}}$ is non-decreasing. By the assumption for $t=1$ we have

$$
\left\|f-h+t\left(h-g_{0}\right)\right\|_{\alpha}^{-^{2}}-\|f-h\|_{\alpha}^{-^{2}} \leq 0 .
$$

Since $\|f-h\|_{\alpha}^{+} \geq\|f-h\|_{\alpha}^{-}$we get

$$
\left\|f-h+t\left(h-g_{0}\right)\right\|_{\alpha}^{-^{2}}-\|f-h\|_{\alpha}^{+^{2}} \leq 0 .
$$

So dividing by $t_{m}, m \rightarrow \infty$, and taking limsup we obtain

$$
\tau_{2}\left(f-h, h-g_{0}\right)_{\alpha}^{-} \leq 0
$$




\section{References}

[1] T. Bag and S.K. Samanta, Finite dimentional fuzzy normed linear spaces, J. Fuzzy Math., 11, No 3 (2003), 678-705.

[2] Y.E. Bao, C.X. Wu, Convexity and semicontinuity of fuzzy mappings, Comput. Math. Appl., 51 (2006), 1809-1816.

[3] S.C. Cheng and J.N. Mordeson, Fuzzy linear operator and fuzzy normed linear spaces, Bull. Calcutta Math. Soc., 86 (1994), 429-436.

[4] J. Fang, H. Huang, On the level convergence of sequence of fuzzy numbers, Fuzzy Sets and Systems, 147 (2004), 417-435.

[5] C. Felbin, Finite dimensional fuzzy normed linear space, Fuzzy Sets and Systems, 48 (1992), 239-248.

[6] C. Felbin, The completion of a fuzzy normed linear space, J. Math. Anal. Appl., 174, No 2 (1993), 428-440.

[7] C. Felbin, Finite dimensional normed linear space II, J. Anal., 7 (1999), 117-131.

[8] R. Goetschel, W. Voxman, Elementary fuzzy calculus, Fuzzy Sets and Systems, 18 (1986), 31-42.

[9] A. Hasankhani, A. Nazari, M. Saheli, Some properties of fuzzy Hilbert spaces and norm of operators, Iranian Journal of Fuzzy Systems, 7, No 3 (2010), 129-157.

[10] O. Kaleva, S. Seikala, On fuzzy metric spaces, Fuzzy Sets and Systems, 12 (1984), 215-229.

[11] A.K. Katsaras, Fuzzy topological vector spaces II, Fuzzy Sets and Systems, 12 (1984), 143-154.

[12] I. Kramosil, J. Michelek, Fuzzy metric and statistical metric spaces, Kybernetica, 11 (1975), 326-334.

[13] S.V. Krishna, K.K.M. Sarma, Separation off uzzy normed linear spaces, Fuzzy Sets and Systems, 63 (1994), 207-217.

[14] Y. Syaua, L. Sugianto, E.S. Lee, A class of semicontinuous fuzzy mappings, Applied Mathematics Letters, 21 (2008), 824-827. 
[15] O. Talo, Some properties of limit inferior and limit superior for sequences of fuzzy real numbers, Information Sciences, 279 (2014), 560-568.

[16] S. M. Vaezpour, F. Karimi , t-Best approximation in fuzzy normed spaces, Iran. J. Fuzzy Syst., 5, No 2 (2008), 93-99.

[17] P. Veeramani, Best approximation in fuzzy metric spaces, J. Fuzzy Math., 9, No 1 (2001), 75-80.

[18] J.-Z. Xiao, X.-H. Zhu, Fuzzy normed spaces of operators and its completeness, Fuzzy Sets and Systems, 133, No 3 (2003), 389-399. 